\title{
ANALYSIS OF THROMBOTIC COMPLICATIONS AFTER CATHETER ABLATION
}

\author{
Luděk Haman ${ }^{1,2}$, Petr Pař́zek ${ }^{1}$, Radovan Malý ${ }^{1}$, Jiří Duda ${ }^{1}$, Jaroslav Malý3
}

\begin{abstract}
Charles University in Prague, Faculty of Medicine in Hradec Králové and University Hospital Hradec Králové, Czech Republic: $1^{\text {st }}$ Department of Internal Medicine ${ }^{1}, 2^{\text {nd }}$ Department of Internal Medicine ${ }^{3}$; University of Defence in Brno, Faculty of Military Medicine in Hradec Králové, Czech Republic: Department of Military Internal Medicine ${ }^{2}$
\end{abstract}

Summary: Introduction: Thromboembolic complications are described in about $1 \%$ of the patients undergoing radiofrequency catheter ablation (RFA). The aim of this study was to analyze thrombotic complications after RFA and to determine prothrombotic states in patients with thrombotic complications. Methods: We analyzed data from 400 patients (212 females) who underwent 453 RFA procedures for supraventricular tachycardias. Transthoracic echocardiography was performed one day before and after RFA in all patients. We evaluated the clinical and laboratory (in patients with thrombotic complications after RFA) risk factors of thromboembolism. Results: We observed thrombotic complication in 7 (1.75\%) patients ( 6 females), thrice flail thrombus in the right atrium, flail thrombus in the inferior vena cava, femoral vein thrombosis with massive pulmonary embolism, femoral vein mural thrombus and upper extremity digital arteries embolization; four of them were asymptomatic. As a prothrombotic state we identified factor V Leiden mutation in one case and the use of oral contraceptives in two cases. Two other patients had a positive history of thromboembolic events. In a subgroup of females the use of oral contraceptives $(p=0.13)$ or a positive history of thromboembolism $(p=0.21)$ were not identified as important risk factors. Conclusion: Echocardiographic detection of asymptomatic thrombotic complications contributed to the higher percentage of these complications in our study. Although we can identify the risk factor (laboratory or clinical) in a majority of patients with a thromboembolic complication, occurrence of these complications is unpredictable.

Key words: Catheter ablation; Thrombosis; Thromboembolism; Complication

\section{Introduction}

Catheter ablation therapy can be performed effectively and safely and can be offered in cases of supraventricular tachycardias as a first-choice therapy. Complications associated with the ablative procedure occur at a low incidence of $2 \%$ to $5 \%(3,4,7,8,9)$. Thromboembolism is a rare, but possible critical complication of radiofrequency catheter ablation (RFA), with an incidence of between $0.6 \%$ to $1.3 \%$ with the risk being higher when the ablation procedure is performed in the left heart (1.8-2\%) and for ventricular tachycardia (2.8\%) (9). However, the published series have dealt primarily with symptomatic complications. The incidence and clinical importance of asymptomatic thromboembolic complications are not well known. Also, the determination of prothrombotic states in patients with thrombotic complications has not yet been studied in detail.

\section{Methods}

\section{Patient characteristics}

The group consisted of 400 patients who underwent 453 RFA procedures for supraventricular tachycardias in our electrophysiology laboratory during a five-year period

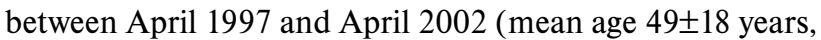
212 (53\%) females).

The arrhythmia types included: AV nodal reentry tachycardia (AVNRT), AV reentry tachycardia (AVRT) caused by accessory AV pathways (AP) either concealed or manifest, clockwise or counterclockwise atrial flutter (FLS), focal or macro reentrant right atrial tachycardia (AT) and AV junction (AVJ) ablation for ventricular rate control in atrial fibrillation (Table 1).

\section{Electrophysiological and $R F$ ablation procedure}

Each patient was studied in the postabsortive state without any sedation. Cardiac antiarrhythmic agents were discontinued for at least 5 half-lives before the study.

Four introducer sheaths ( 6 or $7 F$ ) were positioned in the right and left femoral veins and left subclavian vein. In the patients who required an arterial approach, a 7F sheath was introduced into the right femoral artery. Standard electrophysiology catheters (St Jude Medical, Daig Division, USA; Biosense Webster, USA) were introduced into the coronary sinus, high right atrium, right ventricle and His bundle positions. 
Tab. 1: Demographic and clinical data.

\begin{tabular}{|l|c|c|c|}
\hline & $\begin{array}{c}\text { Total } \\
\text { group }\end{array}$ & $\begin{array}{c}\text { Thrombosis } \\
\text { group }\end{array}$ & $\begin{array}{c}\mathrm{p} \\
\text { value }\end{array}$ \\
\hline Number of patients & 400 & 7 & \\
\hline Mean age (yrs) & $49 \pm 18$ & $52 \pm 17$ & 0.66 \\
\hline Age range (yrs) & $16-80$ & $20-72$ & \\
\hline Male gender (\%) & $188(47 \%)$ & $1(14 \%)$ & 0.12 \\
\hline Procedure time (min) & $195 \pm 74$ & $194 \pm 45$ & 0.97 \\
\hline Number of RF pulses & $10 \pm 9$ & $7 \pm 4$ & 0.41 \\
\hline AV nodal reentry & $180(45 \%)$ & $3(42 \%)$ & 1.00 \\
\hline Accessory pathway & $108(27 \%)$ & $2(29 \%)$ & 0.98 \\
\hline Atrial flutter & $72(18 \%)$ & $2(29 \%)$ & 0.61 \\
\hline Atrial tachycardia & $20(5 \%)$ & 0 & 1.00 \\
\hline AV junction & $20(5 \%)$ & 0 & 1.00 \\
\hline
\end{tabular}

Tab. 2: Clinical risk factors of thromboembolism in the female subgroup.

\begin{tabular}{|l|c|c|c|}
\hline & $\begin{array}{c}\text { Total } \\
\text { group }\end{array}$ & $\begin{array}{c}\text { Thrombosis } \\
\text { group }\end{array}$ & $\begin{array}{c}\mathrm{p} \\
\text { value }\end{array}$ \\
\hline Number of patients & 212 & 6 & \\
\hline Positive history & 8 & 1 & 0.21 \\
\hline Oral contraceptives & 23 & 2 & 0.13 \\
\hline
\end{tabular}

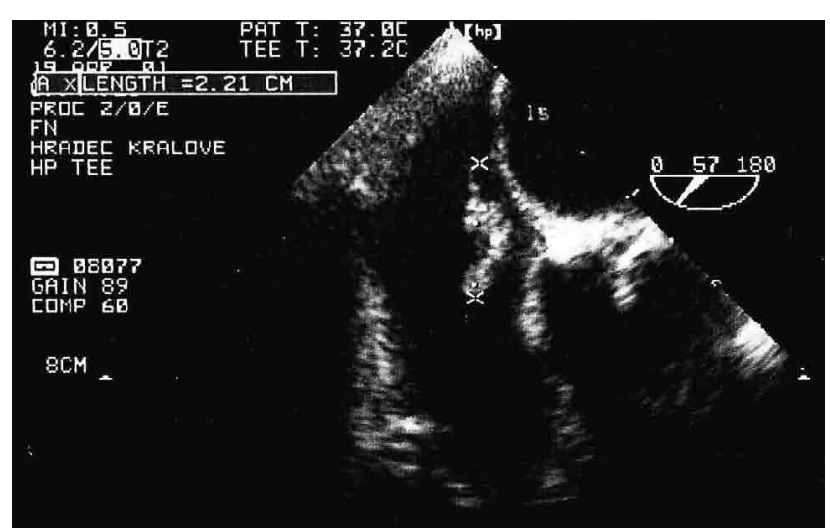

Fig. 1: Flail thrombus attached to the right atrial septum (transesophageal echocardiography picture).

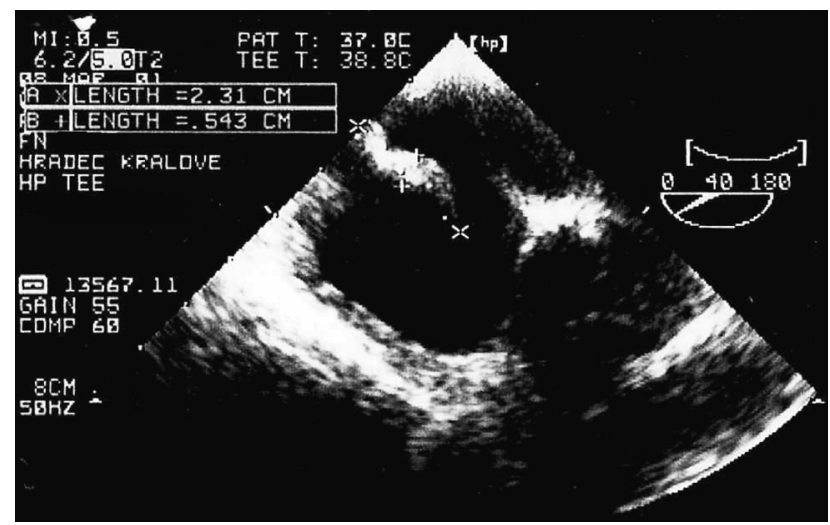

Fig. 2: Flail thrombus in vena cava inferior (transesophageal echocardiography picture).
A detailed electrophysiologic evaluation was performed using standard stimulation and recording techniques to establish the correct diagnosis and to identify the appropriate site for ablation. Radiofrequency energy (temperature controlled) was applied through a $4 \mathrm{~mm}$ tip catheter (St Jude Medical, Daig Division, USA; Biosense Webster, USA); for atrial flutter a $8 \mathrm{~mm}$ tip catheter (St Jude Medical, Daig Division, USA) was used in $20 \%$ of procedures because of impossibility to achieve bidirectional conduction block with standard catheter.

All sheaths were removed immediately after the session was completed. Compression bandage with immobilization was used for 6 hours in case of venous approach, for 24 hours in case of arterial approach.

\section{Anticoagulation regimen}

Intravenous heparin was administered during the procedure only in patients with left-sided RFA procedures. All patients received anticoagulation with LMWH (low-molecular weight heparin) - nadroparinum calcium (Fraxiparine, Glaxo Group Ltd.) subcutaneously, the weight equivalent dose, after the sheaths were removed and on the first and second days after the RFA procedure. Antiplatelet therapy, with $200 \mathrm{mg}$ of aspirin orally, was begun one day after the procedure and continued for 1 month.

\section{Echocardiography}

Transthoracic echocardiography was performed one day before and after RFA in all patients. Intracardiac thrombosis findings were confirmed using transesophageal echocardiography.

\section{Screening of laboratory and clinical risk factors}

In all patients the clinical risk factors of thromboembolism were evaluated (a positive history of the thromboembolic event, malignancy, the use of hormonal therapy, corticosteroid therapy). Laboratory risk factors were evaluated in selected patients with thrombotic complications after RFA procedure (factor $\mathrm{V}$ Leiden, prothrombin G20210A and MTHFR C667T mutations, antithrombin and proteins $\mathrm{C}$ and $\mathrm{S}$ deficiencies, activated protein $\mathrm{C}$ resistance, anticardiolipin antibodies, lupus anticoagulant and plasma homocystein levels).

\section{Statistical analysis}

All data are presented as the mean \pm standard error of the mean. Comparisons between the groups of patients were made with the unpaired Student's t test. Fisher's exact test was performed to compare the clinical findings between the groups. A $P$ value $<0.05$ was considered statistically significant.

\section{Results}

No intracardiac thrombus was observed during preablation echocardiography. 
Thromboembolic complication after RFA was observed in 7 patients $(1.75 \%), 1$ male and 6 females, - thrice flail thrombus in the right atrium, flail thrombus in the inferior vena cava (IVC), femoral vein thrombosis with massive pulmonary embolism, common femoral vein mural thrombus and upper extremity digital arteries embolization with an unknown origin of the thrombosis.

Four of the thrombotic complications - flail thrombi in the right atrium and in the inferior vena cava (Figs 1,2) were symptomless, detected by control echocardiography.

The demographic and clinical data are presented in Tab 1. There were no significant differences in terms of age, procedure time, number of RF pulses or type of treated arrhythmia between the groups.

Laboratory risk factors were analyzed in all patients with thrombotic complications. In one female with a massive pulmonary embolization a factor $\mathrm{V}$ Leiden mutation was identified as a prothrombotic state.

Thrombotic complications were more frequent in the female subgroup - $6(2.8 \%), p=0.12$, including all asymptomatic cases.

In this subgroup we also analyzed the clinical risk factors - a positive history of the thromboembolic event and use of oral contraceptives. Other clinical risk factors (a positive family history, malignancy etc.) were documented rarely.

The clinical data are presented in Tab 2. One female with a flail thrombus in the IVC had a thromboembolic event in her history; two females with flail thrombus in the right atrium and with common femoral vein mural thrombus used oral contraceptives. There were no significant differences in terms of positive history or use of oral contraceptives between the groups.

One serious complication, massive pulmonary embolism, was successfully treated with systemic thrombolytic therapy. The other thrombotic complications were successfully treated with LMWH with complete disappearance of the thrombus after one week of anticoagulation therapy. Anticoagulation therapy was prolonged for 3 months.

During a follow-up (322 \pm 12 months) no late thrombotic complications were observed.

\section{Discussion}

The number of thromboembolic complications in our study is higher then in previously published series $(4,7,8,9)$. The higher incidence of these complications is caused by echocardiographic detection of the asymptomatic thrombi. Calkins et al. (3) described only two thrombotic complications in 972 patients with echocardiograms before and after RFA. Asymptomatic (revealed by control transthoracic echocardiogram) mobile right atrial thrombi were described rarely (2). Chu et al. (5), using intracardiac echocardiography, identified thrombus formation in $19 \%$ of the 81 imaged RF applications; Ren et al. (13) detected small thrombi attached to the transseptal catheter, to the ablation electro- de or to the ablation lesions in 11 patients (20\%). However, the use of intracardiac echocardiography in all patients is not possible.

The mechanism of a so-called soft thrombus formation and the role of the natural coagulation system are unknown. Demolin et al. (6) studied formation of the soft thrombus during experimental ablations - the thrombus formation results from heat induced protein denaturation and aggregation and occurs independently of heparin concentration.

In spite of the unclear clinical importance of asymptomatic thrombotic complications (silent nature of most pulmonary emboli originating in the right heart), the use of a transthoracic echocardiography after the RFA seems to be reasonable.

A precise determination or exclusion of laboratory thromboembolism risk factors in patients with thrombotic complications after RFA has not been previously described. We cannot recommend these very expensive tests for standard screening of patients threatened with thrombosis because of the assumed low prevalence of hereditary or acquired thrombophilia in the RFA patient population. Use of these tests in a selected patient population with thrombotic complications can reveal the cause of thrombosis. Epstein et al. (7) reported an embolic complication in an otherwise risk-free patient in one case out of 830 procedures; risk factors were identified for eight thromboembolic complications (unfortunately comparison with patients without embolic complication is not available). In six females with thromboembolic complication we found a risk factor in four of them (one laboratory and three clinical). In comparison with the "non-complicated group", identification of a clinical risk factor was not sufficient for prediction of possible thromboembolic complications in our study.

Current practice with regard to anticoagulation prophylaxis for catheter ablation procedures varies widely. Our anticoagulation regimen seems to be insufficient for prevention of thrombus formation in all patients. It has been proved, that prolonged administration of heparin (including early administration after the start of the procedure) and/or the association with antiplatelet agents may provide a possible advantage in antithrombotic prevention $(1,10,12)$. Manolis et al. (11) showed that pretreatment of a patient with a combination of aspirin and ticlopidine reduced the D-dimer evidence of thrombosis after ablation. Unfortunately, the available studies included only a small number of patients and we have no scientific evidence that anticoagulant therapies prevent thromboembolism in our patients.

\section{Limitations}

The study is retrospective and underpowered to detect difference in baseline risk factors because of low prevalence of hereditary or acquired thrombophilia in the RFA patient population. 


\section{Conclusion}

Echocardiographic detection of asymptomatic thrombotic complications contributed to the higher percentage of these complications in our study. Although we can identify the risk factor (laboratory or clinical) in a majority of patients with a thromboembolic complication, occurrence of these complications is unpredictable. Further investigation of thrombogenesis during RFA and more extensive studies with thrombin activity inhibitors, direct thrombin inhibitors, platelet activation inhibitors or a combination of these agents will be necessary for standardization of the anticoagulation regimen which will hopefully reduce the risk of thromboembolism after RFA procedures, especially in times of left atrial linear lesions.

\section{References}

1. Anfinsen OG, Gjesdal K, Aass $\mathrm{H}$ et al. When should heparin preferably be administered during radiofrequency catheter ablation? Pacing Clin Electrophysiol 2001;24:5-12.

2. Bosquet $\mathrm{S}$, Sunthorn $\mathrm{H}$, Zaim $\mathrm{S}$ et al. Right atrial thrombus formation after radiofrequency catheter ablation of a left-sided accessory pathway using a transseptal approach. Pacing Clin Electrophysiol 2002;25:1146-8.

3. Calkins H, Yong P, Miller J et al. Catheter ablation of Accessory Pathways, Atrioventricular Nodal Reentrant Tachycardia, and the Atrioventricular Junction: Final Results of a Prospective, Multicenter Clinical Trial. Circulation 1999; 99:262-70.

4. Chen SA, Chiang CE, Tai CT et al. Complications of diagnostic electrophysiological studies and radiofrequency catheter ablation in patients with tachyarrhythmias: An 8-year survey of 3.966 consecutive procedures in a tertiary referral center. Am J Cardiol 1996;77:41-6.
5. Chu E, Kalman JM, Kwasman MA et al. Intracardiac echocardiography during radiofrequency catheter ablation of cardiac arrhythmias in humans. J Am Coll Cardiol 1994;24:1351-7.

6. Demolin JM, Eick OJ, Munch $\mathrm{K}$ et al. Soft thrombus formation in radiofrequency catheter ablation. Pacing Clin Electrophysiol 2002;25:1219-22.

7. Epstein MR, Knapp LD, Martindill M et al. Embolic complications associated with radiofrequency catheter ablation. Atakr Investigators Group. Am J Cardiol 1996;77:655-8

8. Green TO, Huang SK, Wagshal AB et al. Cardiovascular complications after radiofrequency catheter ablation of supraventricular tachyarrhythmias. Am J Cardiol 1994;74:615-7.

9. Hindricks G. The multicenter European Radiofrequency Survey (MERFS): Complications of radiofrequency catheter ablation of arrhythmias. Eur Heart $\mathrm{J}$ 1993; 14:1644-53

10. Lee DS, Dorian P, Downar E et al. Thrombogenicity of radiofrequency ablation procedures: what factors influence thrombin generation? Europace 2001;3: 195-200.

11. Manolis AS, Maounis T, Vassilikos V et al. Pretreatment with antithrombotic agents during radiofrequency catheter ablation: A randomized comparison of aspirin versus ticlopidine. J Cardiovasc Electrophysiol 1998;11:1144-51.

12. Michelucci A, Antonucci E, Conti AA et al. Electrophysiologic procedures and activation of the hemostatic system. Am Heart J 1999;138:128-32.

13. Ren JF, Schwartzman D, Callans DJ et al. Intracardiac echocardiography ( 9 $\mathrm{MHz}$ ) in humans: Methods, imaging views and clinical utility. Ultrasound Med Biol 1999;25:1077-86.

Submitted October 2005.

Accepted March 2006.

MUDr. Luděk Haman, Ph.D., University Hospital Hradec Králové, $1^{\text {st }}$ Department of Internal Medicine, Sokolská 581, 50005 Hradec Králové, Czech Republic. e-mail: haman@fnhk.cz 